\title{
Evaluación fisicoquímica y capacidad antioxidante de moringa (Moringa oleífera) y maracuyá (Passiflora edulis)
}

\author{
Physico-chemical evaluation and antioxidant \\ capacity of moringa (Moringa oleifera) and passion \\ fruit (Passiflora edulis) mps.
}

Nubia-Lisbeth Matute

Universidad Técnica de Machala

(Machala - Ecuador)
Angie Lisseth lópez Universidad Técnica de Machala (Machala - Ecuador)

Ana-Paola Echavarría Universidad Estatal de Milagro (Milagro - Guayas - Ecuador) aechavarriav@unemi.ed.ec
Revista Cumbres Vol.4 №1

Versión impresa ISSN 1390-9541

Versión electrónica ISSN 1390-3365

http://investigacion.utmachala.edu.ec/revistas/index.php/Cumbres 


\title{
RESUMEN
}

La presente investigación tuvo como objetivo evaluar los componentes nutricionales, fisicoquímicos y la capacidad antioxidante de una mezcla óptima a base de maracuyá (Passiflora edulis) y extracto hidroalcohólico de moringa (Moringa oleífera), en diferentes concentraciones (1:2, 1:5 y 1:8), se ha tomado como materia de estudio a estas dos variedades agrícolas con la finalidad de obtener una base para el desarrollo de una bebida funcional. Para definir las propiedades fisicoquímicas (solidos totales, $\mathrm{pH}$, acidez densidad, fructosa y glucosa) de las mezclas se realizó un diseño experimental D-óptimo con las tres concentraciones de mezclas. De acuerdo a los datos obtenidos para la actividad antioxidante y utilizando el método de DPPH (2-difenil-1-picril hidrazilo), se obtuvo una mezcla óptima (1:8), con un valor de IC50 de 139,531 $\mu \mathrm{g} /$ $\mathrm{mL}$ con presencia de saponinas, fenoles y taninos permitiendo así relacionar los resultados obtenidos con su actividad antioxidante.

Palabras clave: Bebida funcional, DPPH, antioxidante, mezcla óptima

\begin{abstract}
The purpose of this study was to evaluate the nutritional, physical and chemical components, and antioxidant capacity of an optimum mixture based on passion fruit (Passiflora edulis) and hydroalcoholic extract of Moringa (Moringa oleifera), in different concentrations (1: 2, 1: 5 and 1: 8). These two agricultural varieties have been taken as the subjects of the study for obtaining a base to develop a functional beverage. To define the physicochemical properties (total solids, pH, acidity, density, fructose, and glucose) of the mixtures, a D-optimal experimental design was performed with three concentrations of mixtures. According to the data resulting from the antioxidant activity using the DPPH (2-diphenyl-1-picrylhydrazyl) method, an optimum 1: 8 concentration was obtained with an IC50 value of $139.531 \mu \mathrm{g} / \mathrm{mL}$. The presence of saponins, phenols, and tannins allowed to relate the results with their antioxidant activity. The results of the study demonstrate that the proposed hypothesis is true because when analyzing the components separately and in the three types of concentrations, an optimum mixture with antioxidant properties was obtained which will serve to develop the functional beverage proposed.
\end{abstract}

Keywords: Optimum mixture, DPPH antioxidant, functional beverage

\section{INTRODUCCIÓN}

Los alimentos funcionales son aquellos que, además de satisfacer las necesidades nutricionales básicas, proporcionan beneficios para la salud o reducen el riesgo de sufrir enfermedades, trasformando la materia prima en produc-

\section{$2 \quad$ Cumbres}


tos alimenticios sanos, nutritivos y seguros, con unas propiedades físico-químicas que prolonguen su vida media y que mantengan o mejoren sus características organolépticas hasta el momento de su consumo (Ashwell, 2002). El desarrollo de alimentos funcionales requiere, además, la presencia de al menos un componente funcional, que debe ser optimizado, añadido o eliminado del alimento (Palou y Serra, 2000). Una de las principales características de un alimento funcional es su capacidad antioxidante (Del Castillo et al, 2005). Los antioxidantes son conocidos por proveer beneficios a la salud, como reducción de riesgo de enfermedades cardiovasculares y cáncer, al combatir el daño celular causado por los radicales libres (Naranjo et al, 2011), los cuales son sustancias químicas muy reactivas que introducen oxígeno en las células y producen la oxidación de sus diferentes partes, alteraciones en el ADN y cambios diversos que aceleran el envejecimiento del cuerpo (Ramírez, et al., 2012).

Las frutas y vegetales poseen propiedades que ayudan a reducir de manera considerable las enfermedades crónicas, como cáncer o enfermedades cardiovasculares (Rojas y Tomás, 2010). Este efecto de protección ha sido asociado con una variedad de constituyentes nutrientes y no nutrientes, siendo muchos de ellos caracterizados por su capacidad antioxidante (Zamora, 2007). Los flavonoides son la subclase de polifenoles más grande y abundante del mundo vegetal. Se distribuyen en las plantas vasculares de manera ubicua y la variedad de sus propiedades biológicas ha llamado poderosamente la atención de los investigadores, de modo que, hoy día, es el grupo de polifenoles más estudiado (Rojas, 2010). Se han descrito para los flavonoides propiedades antioxidantes, antiinflamatorias, antivirales entre otras. El maracuyá (Passiflora edulis) contiene componentes que benefician a la salud, los cuales pueden ser atribuidos a sus macronutrimentos (vitaminas y minerales), como otras frutas exóticas el maracuyá proporciona una significante fuente de nutrimentos (Amaya, 2009). En el análisis de jugos de frutas la licuefacción enzimática es un proceso de hidrólisis de sustratos específicos que componen la pulpa de las frutas, como la pectina, almidón, celulosa y hemicelulosa, de tal manera que clarifican y disminuyen la turbidez y viscosidad indeseada de un proceso de filtración, mejorando el color, aroma y sabor del producto final (Crisostomo et al, 2015)

Investigaciones anteriores han demostrado que las hojas de moringa poseen propiedades antioxidantes al igual que son una fuente excepcional de provitamina A, vitaminas B y C, minerales (hierro) y aminoácidos como la metionina (Met) y L-cistina que contienen azufre; y la leucina (Leu) utilizada por las células para sintetizar las proteínas (Price, 2000).

Este trabajo tiene como objetivo evaluar la formulación de una mezcla compuesta de extractos moringa (Moringa oleífera) y maracuyá (Passiflora edulis) como base para el desarrollo de una bebida funcional.

\section{MATERIALES Y MÉTODOS}

Las hojas de Moringa oleífera se recolectaron de la unidad académica de Agronomía (UTMACH) y los frutos de maracuyá (Pasiflora edullis) en el Cantón 
Santa Rosa (Provincia del Oro). Las muestras utilizadas se limpiaron y lavaron con agua destilada, se secaron en estufa a 30oC y posteriormente se molieron en un triturador de cuchillas. Una vez obtenida la muestra seca y pulverizada se procede a macerar en solución etanólica (1:1) al 98\%, dejando reposar en un erlenmeyer durante 42 horas. El extracto obtenido de la filtración se concentró llevándolo a un rotavapor a presión reducida, para reducir su volumen y evaporar el solvente, quedando como resultado un extracto no volátil.

Se seleccionaron muestras del fruto de maracuyá sin daños externos las cuales pasaron por un proceso de lavado por inmersión en agua potable, se procedió a extraer la pulpa del fruto, para ello, se utilizó la enzima Rapidase ${ }^{\circledR}$; donde se tomó $10 \mu \mathrm{l}$ de enzima y se le adicionó a 74 g de muestra, se dejó reposar durante 10 min. A continuación, se filtró con papel filtro No 4, obteniéndose la pulpa extraída del maracuyá.

El análisis se realizó con una extracción hidroalcohólica de las hojas de moringa (soluto) y el extracto acuoso de la pulpa de maracuyá como solvente, de las mezclas estudiadas se prepararon tres diluciones (1:2 1:5 1:8) expresadas en vlv. Para determinar la viscosidad de las muestras se utilizó un viscosímetro rotacional Fungilab, con el manejo del husillo Ll y una velocidad de corte de $100 \mathrm{rpm}$. El índice de madurez se determinó como la relación entre los sólidos solubles y la acidez titulable y fue expresado como ${ }^{\circ} \mathrm{Brix} / \mathrm{g}$ ácido cítrico. Los sólidos solubles se midieron sobre la pulpa del fruto con un refractómetro Abbemat 200 y se expresaron como ${ }^{\circ}$ Brix. La acidez titulable se midió mediante una titulación con una solución estandarizada de $\mathrm{NaOH}$ 0,1 N. La acidez titulable se expresó en g de ácido cítrico.

El diseño experimental fue completamente al azar de tres niveles, se utilizó el programa estadístico Statgraphics v.5.0., en donde se midieron las variables de las muestras a diferentes concentraciones para determinar el valor óptimo de las mezclas.

Se evaluaron los principales componentes físicos (humedad, cenizas, densidad, viscosidad, $\mathrm{pH}$, acidez, sólidos solubles) y químicos presentes en las especies estudiadas. Los ensayos de saponinas y taninos se realizaron siguiendo la metodología sugerida por De Astudillo et al. (2015), con algunas modificaciones. La actividad antioxidante de los extractos se evaluó mediante la capacidad captadora del radical DPPH • (1,1-difenil-2-picril hidracilo), utilizando la metodología de Echavarria et al. (2013). Un volumen de 990 $\mu \mathrm{L}$ de una solución metanólica de DPPH se mezcló con $10 \mu \mathrm{L}$ de las soluciones hidro-etanólicas (50:50) de cada extracto a varias concentraciones; las mezclas se dejaron en reposo y en ausencia de luz durante 30 minutos, se leyó la absorbancia a $517 \mathrm{~nm}$ en un espectrofotómetro UV-Visible (Shimadzu, UV mini -1240). Los resultados fueron convertidos a porcentaje de inhibición y expresados como capacidad antioxidante en $\mu \mathrm{mol}$ de equivalentes Trolox (ácido-6-hidroxi-2, 5, 7,8-tetrametilcroman-2-carboxílico); TE)/g de EEP (TEAC). Los experimentos se llevaron a cabo usando un bloque de diseño al azar. Dentro de cada bloque cada tratamiento se aplicó 3 veces. Se usó como control positivo ácido ascórbico. 


\section{RESULTADOS Y DISCUSIÓN}

El promedio del maracuyá utilizado fue de $160 \mathrm{~g}$ de peso, longitud de 7,53 $\mathrm{cm}$ y diámetro de 7,03 cm. Las hojas de moringa pesaron $48 \mathrm{~g}$ con un rendimiento favorable debido a que presentaron un bajo contenido de humedad.

En la Tabla 1, se observa el porcentaje de fructosa en las muestras analizadas a diferentes concentraciones, siendo el porcentaje más elevado el de la mezcla 1:8 (23,21\%), presentando una relación con el extracto puro de moringa (Moringa oleífera), que contiene un valor de $22,60 \%$, el concentrado de maracuyá (Passiflora edulis) presentó un valor de 13,24 \%. Del mismo modo, el contenido de glucosa de la muestra de moringa mostró un valor alto de $22,39 \%$.

Tabla 1. Fructosa y glucosa de las muestras expresadas en cantidades porcentuales

\begin{tabular}{|l|c|c|}
\hline \multicolumn{1}{|c|}{ Muestra } & Fructosa (\%) & Glucosa (\%) \\
\hline Maracuyá & $13,82 \pm 0,26$ & $13,24 \pm 0,22$ \\
\hline Moringa & $22,60 \pm 0,10$ & $22,39 \pm 0,22$ \\
\hline C 1:2 & $19,12 \pm 0,10$ & $18,64 \pm 0,10$ \\
\hline C 1:5 & $19,68 \pm 0,03$ & $18,95 \pm 0,01$ \\
\hline C 1:8 & $23,21 \pm 0,01$ & $23,00 \pm 0,01$ \\
\hline
\end{tabular}

Los valores de las propiedades físicas de las muestras iniciales y mezclas a diferentes concentraciones se aprecian en la Tabla 2.

La densidad se ve afectada directamente por el contenido de extracto de maracuyá en cada una de las concentraciones, puesto que en la de menor concentración (1:2) presenta una densidad de 1,054 g/mL, mientras que aquella que posee mayor contenido de maracuyá (1:8) presentó una densidad de $1,043 \mathrm{~g} / \mathrm{mL}$.

Tomando en consideración valores de las muestras y las concentraciones 1:2, 1:5 y 1:8 de las mezclas para la determinación de la viscosidad se obtuvieron valores entre $60 \mathrm{mPas}-40 \mathrm{mPas}$, al igual que las otras propiedades como $\mathrm{pH}$, acidez, que se encuentran en el rango establecido por las normas INEN ( 2 337:2008) para jugos de frutas. 
Tabla 2. Determinación de las propiedades físicas de las muestras

\begin{tabular}{|c|c|c|c|c|c|c|}
\hline Muestra & $\mathrm{pH}$ & $\begin{array}{c}\text { Acidez } \\
(\mathbf{g} / \mathbf{1 0 0 g})\end{array}$ & $\begin{array}{c}\text { S. solubles } \\
\text { oBrix }\end{array}$ & $\begin{array}{c}\text { Índice de } \\
\text { refracción } \\
\mathbf{( N d )}\end{array}$ & $\begin{array}{c}\text { Densidad } \\
(\mathbf{g} / \mathbf{m L}\end{array}$ & $\begin{array}{c}\text { Viscosidad } \\
\text { (mPas) }\end{array}$ \\
\hline Maracuyá & $3,12 \pm 0,03$ & $0,57 \pm 0,02$ & $13,76 \pm 0,01$ & 1,35 & 1,066 & 55 \\
\hline Moringa & $5,60 \pm 0,03$ & $0,53 \pm 0,02$ & $22,29 \pm 0,01$ & 1,37 & 1,040 & 30 \\
\hline C $1: 2$ & $3,33 \pm 0,03$ & $0,56 \pm 0,02$ & $19,42 \pm 0,02$ & 1,36 & 1,054 & 42 \\
\hline C 1:5 & $3,48 \pm 0,02$ & $0,55 \pm 0,02$ & $19,63 \pm 0,02$ & 1,37 & 1,049 & 40 \\
\hline C 1:8 & $3,74 \pm 0,02$ & $0,54 \pm 0,03$ & $23,13 \pm 0,01$ & 1,37 & 1,043 & 38 \\
\hline
\end{tabular}

Los resultados del análisis fitoquímico de las muestras se presentan en la Tabla 3, con la presencia y ausencia de familias de metabolitos secundarios como: flavonoides, taninos, saponinas y compuestos cianogénicos en las mezclas analizadas. En la muestra C (1:8) el contenido de flavonoides fue positivo pasando de un color amarillo a naranja intenso. Las saponinas presentaron espuma abundante y estable al realizar el ensayo de Grignard (Mena, 2015). En la pulpa de maracuyá y las hojas de moringa se observaron niveles muy bajos de glucósidos cianogénicos (Rojas et al, 2006).

Tabla 3. Determinación cualitativa de flavonoides, fenoles, saponinas y compuestos cianogénicos

\begin{tabular}{|l|l|l|l|l|l|}
\hline \multirow{2}{*}{ Compuestos } & \multicolumn{5}{|c|}{ Muestras } \\
\cline { 2 - 7 } & Maracuyá & Moringa & C 1:2 & C 1:5 & C 1:8 \\
\hline flavonoides & + & - & + & - & - \\
\hline taninos y Fenoles & + & ++ & + & + & ++ \\
\hline saponinas & - & + & - & + & + \\
\hline g. cianogénicos & - & - & - & - & - \\
\hline
\end{tabular}

\section{CONCLUSIONES}

De acuerdo a los datos obtenidos en los diferentes análisis, la mezcla óptima fue la de concentración 1:8, la cual obtuvo un valor de 050 de 139,531 $\mu \mathrm{g} / \mathrm{mL}$ y 42,4 \% de inhibición muy cercano al del ácido ascórbico (44%). Se detectó la presencia de saponinas, taninos y fenoles, que al igual que su actividad antioxidante, sugieren la posibilidad de elaborar una bebida funcional con las mezclas estudiadas.

\section{REFERENCIAS BIBLIOGRÁFICAS}

Amaya, J. (2009). El cultivo del maracuyá (Passiflora edulis form. Flavicarpa). Gerencia Regional Agraria La Libertad, Trujillo-Perú. 30p. 
Ashwell, M. (2002). Conceptos sobre los alimentos funcionales. International Life Sciences Institute. 83 (6). 42-45 p. ISBN: 1-57881-157-0.

Cortés, A. (2004). Aplicación de enzimas en la producción industrial. Mundo alimentario. (62:10). 22 p.

Crisóstomo, O. Encina, C. Aguirre, R. Silva, B. (2015). Evaluación del proceso de licuefacción enzimática de arilos de pulpa de maracuyá (Passiflora edulis Simms, var. flavicarpa). Revista Peruana de Química. (18: 1) 81-89.

De Astudillo. L, Jaramillo, C. Lemus M. Primera edición 2015. Métodos analíticos para la determinación de metabolitos secundarios. Machala, Ecuador Ediciones UTMACH. ISBN: 978-9978-316-62-7. 51-66 p.

Del Castillo, M. Gordon, M. Ames, J. (2005). Peroxyl radical-scavenging activity of coffee brews. European Food Research Technology. (221):471-7.

Echavarría, AP, Pagan, J y Ibarz, A. (2013). Optimization of Maillard reaction products isolated from sugar-amino acid model system and their antioxidant activity Afinidad LXX (70) 562.

Mena, L. Tamargo, B. Salas, E. Plaza, L. Blanco, Y. (2015). Determinación de saponinas y otros metabolitos secundarios en extractos acuosos de Sapindus saponaria L. (jaboncillo). Revista Cubana de Plantas Medicinales (20-1)106-116.

Naranjo, M. Vélez, L Y Rojano, B. (2011). Actividad antioxidante de café colombiano de diferentes calidades. Revista Cubana de Plantas Medicinales, 16(2), 164-173.

NTE INEM 2 337:2008. Normas INEN para jugos de frutas. Quito-Ecuador.

Palou, F. Serra. (2000). Perspectivas europeas sobre los alimentos funcionales. Alimentación Nutrición y Salud: (7:3) 76 - 90p

Price, M. (2000). The Moringa tree. Educational concerns for hunger Organization (ECHO). Technical Note.

Ramírez, J. García, C. Vizcaíno, J. Cárdenas, J. Gutiérrez, F. (2012). ¿Qué son y para qué sirven los antioxidantes? Revista de divulgación científica y tecnológica de la Universidad Veracruzana, XXV (2).

Rojas, B. (2010). Diseño y evaluación de una bebida funcional con bifidobacterias a partir de pérsimo. Instituto Politécnico Nacional, Mexico, D.F. 84p.

Rojas, J y Tomás G. (2010). Tamizaje fitoquímico y actividad antioxidante in 
vitro de Passiflora edulis sims (Maracuyá). Revista. Peruana. (13:1). 23- 29p

Rojas, J. Ronceros, S. Palomino, R. Tomás, G. (2006). Efecto antihipertensivo y dosis letal 50 del jugo del fruto y del extracto etanólico de las hojas de Passiflora edulis (maracuyá), en ratas. Anales de la Facultad de medicina. (67:3) 206-213.

Zamora, J. (2007). Antioxidantes: micronutrientes en lucha por la salud. Revista chilena de nutrición, 34(1), 17-26. 